\title{
P-TEFb Inhibitor BAY1143572
}

National Cancer Institute

\section{Source}

National Cancer Institute. P-TEFb Inhibitor BAY1143572. NCI Thesaurus. Code C121456.

An inhibitor of positive transcription elong ation factor b (P-TEFb), which is composed of cyclin-dependent kinase 9 (CDK9) and cyclin-T (CycT), with potential antineoplastic activity. Upon administration, BAY1143572 binds to and inhibits the activity of P-TEFb, thereby preventing the phosphorylation of its downstream target, the carboxyl terminal domain (CTD) of RNA polymerase II (RNA Pol II), and inhibiting the activation of transcriptional elong ation by RNA Pol II. This prevents the transcription of tumor promoting genes, induces tumor cell apoptosis, and inhibits tumor cell proliferation. PTEFb plays an important role in the regulation of gene transcription; over-activation in cancer cells leads to both the transcription of key tumor-promoting genes and cancer cell proliferation. 